

\title{
High-Brightness Injectors for Hadron Colliders
}

\author{
Thomas P. Wangler \\ Accelerator Technology Division, MS-H817 \\ Los Alaunos National Laboratory, Los Alamos, NM 87545 USA
}

\section{Introduction}

The counterrotating beams in collider rings consist of trains of beam bunches with $\mathrm{N}_{B}$ particles per bunch, spaced a distance $S_{B}$ apart. When the bunches collide, the interaction rate is determined by the luminosity, which is defined as the interaction rate per unit cross section. For head-on collisions between cylindrical Gaussian beams moving at speed $\beta c$, the luminosity is given by

$$
L=\frac{N_{B}^{2} \beta c}{4 \pi \sigma^{2} S_{B}}
$$

where $\sigma$ is the rms beam size projected onto a transverse plane (the two transverse planes are assumed identical) at the interaction point. This beam size depends on the rms emittance of the beam and the focusing strength, and is given by

$$
\sigma=\left[\frac{\varepsilon_{n} \tilde{\beta}^{*}}{\beta \gamma}\right]^{1 / 2},
$$

where $\varepsilon_{n}$ is the transverse normalized rms emittance, which is a measure of the 2-D phasespace aras in each transverse plane, and is defined in terms of the second moments of the beam distribution. Our convention is to use the true rms normalized emittance, without factors of 4 or 6 that are sometimes used. The quantity $\tilde{\beta}^{*}$ is the Courant-Snyder betatron amplitude function at the interaction point, a characteristic of the focusing lattice, and $\gamma$ is the relativistic Lorentz factor. Using this expression for $\sigma$, the luminosity becomes

$$
L=\frac{N_{B}^{2} \gamma \beta^{2} c}{4 \pi \tilde{\beta}^{*} \varepsilon_{n} S_{B}} .
$$

Achieving high luminosity at a given energy, and at practical values of $\tilde{\beta}^{*}$ and $S_{B}$, requires a large value for the ratio $N_{B}^{2} / \varepsilon_{n}$, which implies high intensity and small emittance. Thus, specification of the luminosity sets the requirements for beam intensity and emittance, and establishes the requirements on the performance of the injector to the collider ring. In general, for fixed $N_{B}$, the luminosity can be increased if $\varepsilon_{n}$ can be reduced.

The minimum emittance of the collider is limited by the performance of the injector; consequently the design of the injector is of great importance for the ultimate performance of the collider. As a numerical example of luminosity that one hopes to achieve, we consider the SSC design. ${ }^{1}$ Assuming $N_{B}=0.75 \times 10^{10}$ protons per bunch, $\gamma=21 \times 10^{3}, \beta=1, \tilde{\beta}^{*}=0.5 \mathrm{~m}, \varepsilon_{n} \pi=1 \times 10^{-6} \pi \mathrm{m}$-rad, $S_{B}=5 \mathrm{~m}$, we obtain $\sigma=4.9 \times 10^{-6} \mathrm{~m}$, and $\mathrm{L}=1.1 \times 10^{33} \mathrm{~cm}^{-2} \mathrm{~s}^{-1}$. 


\subsection{Injector System Architecture}

The injector system for a collider is itself a major high-energy accelerator facility. It consists of a dc injector, an $\mathrm{rf}$ linac, and a multistaged synchrotron accelerator. There are practical reasons for this sequence of accelerators, each of which is ideal within a given energy range, which allows the beam energy to be raised by orders of magnitude while maintaining high intensity and low beam emittances. As will be discribed later, the emittances can grow for a variety of reasons specific to each individual section, and for maximum luminosity the emittance growth in each of these sections must be carefully controlled.

The $\mathrm{H}^{-}$beam for a p-p collider originates in the ion source, from which the beam is extracted and injected into the linac for further acceleration. $\mathrm{H}^{-}$ions are chosen because, using multiturn injection through a stripper foil into a synchrotron ring, ${ }^{2}$ a large circulating current of $\mathrm{H}^{+}$ions can be forned by the overlaying of many turns into the same phasespace area. Because many turns can be used to fill the ring, the procedure does not require a large current in the ion source and linac, and this reduces the linac space-charge problems. The output beam from the linac can be allowed to debunch, and subsequently be rebunched after injection into the ring at an if frequency unrelated to the linac frequency. Alternatively the bunch structure from the linac can be maintained, and the bunches can be injected into preexisting buckets in a ring, whose rf frequency is synchronized with that of the linac. After acceleration to full energy in the lowest energy ring, the beam is extracted in a single turn, transported, and injected into the next ring, which has a larger circumference, consistent with it's higher output energy. The process is repeated, and beams can be stacked end-to-end until the second ring is full. Then acceleration of the $\mathrm{H}^{+}$beam in the second ring brings the beam to the full energy of the second ring, where it can be single-turn extracted and transferred to the next ring. This process of stacking and transferring beams continues until both collider rings are filled with beams from the injector system.

The advantages of using a multistage synchrotron for a high-brightness injector ${ }^{3}$ include two technical reasons: 1) reduced rf frequency range for each ring, and 2) avoidance of low-field operation of the magnets, where the field quality is not as good. Also, an important fundamental reason for a multistage design is the space-charge force, which causes a spread in the transverse (betatron) single-particle oscillation frequencies (tunes) and can result in excitation of beam resonances, with consequent growth of emittance and loss of beam. The effect of the space-charge forces is to defocus the beam and consequently to reduce the net focusing, or cause a downward shift of the transverse tune. The expression for the space-charge-induced tune shift ${ }^{4,5}$ is

$$
\Delta \nu=\frac{-r_{p} N_{T}}{4 \pi \varepsilon_{n} \gamma^{2} \beta} \frac{F G H}{\hat{B}},
$$

where $r_{p}=e^{2} / 4 \pi \varepsilon_{0} m c^{2}\left(1.535 \times 10^{-18} m\right.$ for the proton $), N_{T}$ is the total number of particles in the ring, $F$ describes the image forces from the vacuum chamber and magnet poles ( $F \approx 1$ when image terms can be neglected), $G$ describes the transverse density distribution ( $G=1$ for uniform, 2 for parabolic, and 1.56 for Gaussian beams), and $H$ is a correction for an elliptical beam ( $H=1 / 2$ for a circular beam). The quantity $\hat{B}$ is a bunching factor, the ratio of bunch length to bunch spacing (smaller $\hat{B}$ corresponds to more bunching). For further discussion we will assume a circular parabolic beam, whose image forces can be ignored; then $F G H=1$. For a ring with circumference $C$, we may write $N_{T}=N_{B} C / S_{B}$. 
Substituting this into Eq. (1.4) gives

$$
\Delta \nu=\frac{-r_{P} N_{B} C}{4 \pi \varepsilon_{n} \gamma^{2} \beta S_{B}} \frac{F G H}{\hat{B}} .
$$

For a given choice of the operating tune, there is a limit on $|\Delta \nu|$ to avoid serious resonances and loss of the beam. For a uniform beam the tune shift for all particles is the same, and the shift could in principle be canceled by increasing the external focusing. For a nonuniform beam distribution, the full tune shift is experienced only by the small-amplitude particles, and $|\Delta \nu|$ also approximately represents the space-charge tune spread of the beam. Then a simple cancellation is not obtained for all the particles and one must restrict $|\Delta \nu|$. The maximum admissible value for $|\Delta \nu|$ depends on the resonances near the beam operating points and their effective widths or stopbands. When a particle tune lies within a stopband, a large growth of the amplitude occurs, leading eventually to loss of the particles. A common rule-of-thumb for design to avoid dangerous resonances is to keep the tune spread below about 0.25 . We see that $|\Delta \nu|$ is largest at the injection (lowest) energy, where $\beta \gamma^{2}$ is smallest. For the SSC low-energy booster, at $600 \mathrm{MeV}$ where $\beta \gamma^{2}=2.13$, we assume $\hat{\beta}=0.25, \varepsilon_{n}=0.6 \times 10^{-6} \mathrm{~m}-\mathrm{rad}, C=540 \mathrm{~m}, S_{B}=5 \mathrm{~m}, N_{B}=$ $1.0 \times 10^{10}, G=1.56$ (Gaussian), $F=1$, and $H=1 / 2$. The tune shift calculated from Eq. (1.4) is $|\Delta \nu|=0.32$, slightly larger than the conventional rule of thumb. Values of $|\Delta \nu|=$ 0.37 at the Fermilab booster, ${ }^{6}$ and $|\Delta \nu|=0.6$ at the CERN booster ${ }^{7}$ have been achieved. These results have shown that some compensation for the effects of the resonances can be obtained experimentally, allowing larger tune shifts and higher brightness performance in the rings.

For given values of $N_{B}, S_{B}$, and $\varepsilon_{n}$, we see from Eq. (1.5) that $|\Delta \nu|$ can be reduced by reducing the ring circumference $C$, or increasing the bunching factor $\hat{B}$, or increasing the injection energy. Increasing the injection energy helps for three reasons: 1 ) reduces the total self force (factor of $\gamma^{2}$ ), because of the larger magnetic self force at higher energies, 2 ) increases relativistic inertia (factor of $\gamma$ ), and 3) increases the revolution frequency corresponding to a stronger focusing force for a given tune $\nu$, because tune is defined as the ratio of betatron frequency to revolution frequency. Larger $\hat{B}$ helps because it reduces the peak current. Reduced circumference helps because it increases the revolution frequency for a given $\nu$.

The bunching factor is determined by the choice of synchronous phase, which also affects the acceleration rate. If $\hat{B}$ is fixed, there are two practical ways of reducing $|\Delta \nu|$, 1 ) increasing the injection energy, and 2) decreasing the ring circumference $C$. The latter choice would be at the expense of reduced final energy (for a given latrice and maximum dipole field). Thus, achieving an acceptable value for $|\Delta \nu|$ can limit both the injection and final energies, and provides a fundamental reason for a multistage synchrotron.

\section{Emittance Concept and Definitions}

Beams are composed of collections of particles, which can be described by six normalized phase-space coordinates $\left(x, \Delta P_{x} / m c, y, \Delta P_{y} / m c, z \Delta P_{z}, / m c\right)$. The longitudinal coordinates can be given in terms of either position and normalized momentum differences relative to a synchronous or reference particle $\left(z, \Delta P_{z} / m c\right)$, or time and kinetic energy differences $(\Delta t, \Delta W)$, or phase and kinetic energy differences $(\Delta \phi, \Delta W)$, with $\Delta \phi=2 \pi c \Delta t / \lambda$, where $\lambda$ is the rf wavelength. In general we can talk about a $6-\mathrm{D}$ phasespace distribution that describes the beam at any location in the accelerator. 
As the beam is transported and accelerated, the distribution obeys Liouville's theorem, ${ }^{8}$ provided that the proper conditions are satisfied. These are that 1) the continuity equation is valid in 6-D phase space, which means that no particles are created or lost, and 2) the forces are derivable from a potential function that depends only on the three spatial coordinates and time. Subject to these requirements the theorem states that the density in 6-D phase space, measured along the trajectory of a particle, is invariant, or equivalently that the 6-D volume enclosed by any isodensity contour is invariant. Only when the motion is uncoupled, so that the three 2-D phase-space projections $\left(x, \Delta P_{x} / m c\right)$, $\left(y, \Delta P_{y} / m c\right)$, and $\left(z, \Delta P_{z} / m c\right)$ are independent, does Liouville's theorem apply separately in each of these projections. ${ }^{9}$

For the more general case where the beam particles interact with each other through their mutual Coulomb forces, the forces are not in principle derivable from a potential function that depends only on the three spatial coordinates. But, in most circumstances for practical beams, these interactions can be accurately described by an average collective potential, arising from the contributions of all the particles, and individual binary Coulomb collisions represent only a small effect. Under these circumstances, Liouville's theorem remains a good approximation. The condition for which the binary Coulomb collisions can be ignored and the self interactions treated as a collective space-charge force, is that the number of particles, $N_{D}$, within a Debye sphere is much greater than unity. ${ }^{10}$ Thus

$$
N_{D}=n \frac{4}{3} \pi \lambda_{D}^{3} \gg 1
$$

where $n$ is the nominal beam density, and nonrelativistically

$$
\lambda_{D}=\sqrt{\varepsilon_{0} k T / n e^{2}}
$$

is the Debye length of the beam, where $k T=m c^{2} \varepsilon_{n}^{2} / a^{2}$. This criterion reflects the idea that the forces on a given particle from binary Coulomb collisions are important only for particles within approximately a sphere of radius equal to $\lambda_{D}$. The perturbing influence of individual particles further away than $\lambda_{D}$ is small because of the shielding effect of the other charges. Thus, particles further than $\lambda_{D}$ contribute only to the longerrange collective force. Within the Debye sphere the effect of individual collisions is also not important, when there are a lot of other particles in the sphere that can exert forces to approximately cancel the force of any single particle. For an equivalent uniform beam, whose total beam radius is twice the rms size of the beam, and for the case of a spherical bunch geometry, we find

$$
N_{D}=\frac{4 \pi}{3 n^{1 / 2}}\left[\frac{\varepsilon_{0} m c^{2}}{e^{2}}\right]^{3 / 2}\left[\frac{\varepsilon_{n}}{a}\right]^{3}
$$

where $n$ is the density and $a$ is the rms beam size in any projection. If the Debye length is larger than the beam radius, all particles in the bunch are contained within a Deylue sphere, and the criterion expressed by Eq. (2.1) is replaced by $N_{B} \gg 1$, where $N_{B}$ is the number of particles in the bunch. For practical high-intensity beams, for example in low-energy linacs, we find that $\lambda_{D} \approx a, N_{D} \gg 1$, and Liouville's theorem is a good approximation. As an example, we consider a bunched $1-\mathrm{MeV}, 25-\mathrm{mA}$ proton beam in a $400-\mathrm{MHz}$ linac, with an $\mathrm{rms}$ normalized emittance of $\varepsilon_{n} \pi=0.2 \pi \mathrm{mm}$-rad, and an $\mathrm{rms}$ beam size of $x_{r m s}=1 \mathrm{~mm}$. The number of particles per bunch is $N_{B}=I \lambda / e c=3.9 \times 10^{8}$, $k T=m c^{2}\left(\varepsilon_{n} / a\right)^{2}=38 \mathrm{eV}$, and the density is $n=1.2 \times 10^{16} \mathrm{~m}^{-3}$. This corresponds to 
$\lambda_{D}=0.42 \mathrm{~mm}$, and $N_{D}=3.7 \times 10^{6} \gg 1$, so that Liouville's theorem should be a very good approximation.

The total emittances for an idealized hard-edged uniform 2-D phase-space distribution in our convention are defined as $1 / \pi$ times the phase-space area. This definition corresponds to the product of the semi-axes of an ellipse, and it is useful because the distributions of beams that are matched when only linear forces are present have concentric elliptical contours. For real beams, however, the phase-space density is usually nonuniform, and rarely are the phase-space boundaries sharp. Also the elliptical contours can become broadened or deformed by processes that violate Liouville's theorem, such as scattering or collimation, and can become deformed by forces that couple the degrees of freedom, by nonlinear forces arising from beam-optical aberrations, or by space-charge forces associated with nonuniform density distributions. For a single degree of freedom the deformation from nonlinear forces takes the appearance of a filamentation of an initial elliptical phase space distribution, and is fundamentally a consequence of dependence of the particle oscillation frequency on variables such as amplitude, energy, etc., so that different particles rotate in phase space at different rates. Unless a beam is properly matched, which is not always possible, these filamentation-type distortions further increase some amplitudes, especially of the outermost particles in phase space, and result in an increase in the average beam size. As the filamentation process continues, the phase-space distribution can take a shape much like a spiral galaxy, as regions devoid of particles are captured within outer spiral arms. Because the average phase-space density including the empty regions decreases, the process is sometimes referred to as emittance dilution. For two and three degrees of freedom with coupled nonlinear forces, the projection of outer filaments onto 2-D phase space plots can create a diffuse outer halo.

The resulting increase in rms beam size can be attributed to an increase in effective emittance of the beam. The magnitude of this effective emittance will depend both on processes that violate Liouville's theorem and on processes that cause distortions and filamentations in the distribution. A convenient definition of an effective emittance that has these properties and is applicable to all distributions, is the normalized rms emittance, which for the $\mathrm{x}$-direction is defined, using the Sacherer definition, ${ }^{11}$ in terms of the second moments of the distribution $\overline{x^{2}}, \overline{p_{x}^{2} /(m c)^{2}}$, and $\overline{x p_{x}} /(m c)$ as

$$
\varepsilon_{n, x}=\left[\overline{x^{2}} \overline{p_{x}^{2}}-{\overline{x p_{x}}}^{2}\right]^{1 / 2} /(m c) .
$$

In the paraxial approximation for the transverse motion, the divergence $x^{\prime}$ is related to the transverse momentum component $p_{x}$ by $p_{x}=m c \beta \gamma x^{\prime}$. Using this result the rms normalized emittance for the transverse planes can be written in a more familiar form as

$$
\varepsilon_{n, x}=\beta \gamma\left[\overline{x^{2}} \frac{}{x^{\prime 2}}-\overline{x x}^{\prime 2}\right]^{1 / 2}
$$

Another convention for emittance, first introduced by Lapostolle ${ }^{12}$, is frequently used, in which the right sides of Eqs. (2.4) and (2.5) are multiplied by 4 ; in this convention the emittance reduces to the total emittance for a beam with a uniform hard-edged distribution in the $x-x^{\prime}$ projection. It has become more common recently to refer to the Lapostolle definition as the 4-rms emittance.

It is easily shown that a straight line in phase space, where $x \propto x^{\prime}$, has zero rms emittance, whereas a nonlinear relation between $x$ and $x^{\prime}$ gives a nonzero value, even 
though the thickness of the line is zero. 'The definitions in Eqs. (2.4) and (2.5) can be further motivated as follows. Consider an arbitrary phase-space distribution, whose second moments are known. Suppose we want to describe the distribution using an average ellipse, whose maximum projections on the displacement and momentum axes are chosen to equal the rms values along these axes. This allows us to relate the second moments of the distribution to the emittance $\varepsilon_{n}$ of the ellipse and to the Courant-Snyder ellipse parameters $\tilde{\alpha}, \tilde{\beta}$, and $\tilde{\gamma}$. Thus we require

$$
\overline{x^{2}}=\varepsilon_{n} \tilde{\beta} / \beta \gamma
$$

and

$$
\overline{x^{\prime 2}}=\varepsilon_{n} \tilde{\gamma} / \beta \gamma
$$

The other parameter $\tilde{\alpha}$ that describes the orientation of the ellipse is kinematically related to the rate of change of the amplitude parameter $\tilde{\beta}$ through the expression $\tilde{\alpha}=-(1 / 2) d \tilde{\beta} / d s$, where $s$ is the beam direction. We also use the result $\overline{d x^{2}} / d s=2 \overline{x x^{\prime}}$, to obtain

$$
\tilde{\alpha}=-\overline{x x^{\prime}} \beta \gamma / \varepsilon_{n} .
$$

The Courant-Snyder parameters for the ellipse are related by $\tilde{\beta} \tilde{\gamma}-\tilde{\alpha}^{2}=1$, and when Eqs. (2.6), (2.7), and (2.8) are substituted into the result, we obtain the definition of rms emittance given by Eq. (2.5).

The usefulness of this rms emittance definition was further confirmed by Sacherer ${ }^{11}$ and Lapostolle, ${ }^{12}$ who showed that the rms beam size $a=\left(\overline{x^{2}}\right)^{1 / 2}$ satisfies an envelope equation of motion, which is almost independent of the distribution. For a 2-D round beam in a uniform focusing channel with a wave number $k_{0}$, this becomes

$$
a^{\prime \prime}+k_{0}^{2} a-\frac{\varepsilon^{2}}{\beta^{2} \gamma^{2} a^{3}}-\frac{K}{4 a}=0,
$$

where $K$ is an intensity parameter called the generalized perveance, to be defined later.

Some useful properties of rms emittance can be obtained by differentiating Eq. (2.5) with respect to the coordinate $s$ along the beam direction. It can then ${ }^{13}$ be shown that

$$
\frac{d \varepsilon_{n}^{2}}{d s}=\frac{2 \gamma^{2}}{m c^{2}}\left[\overline{x^{2}} \overline{x^{\prime} F}-\overline{x x^{\prime}} \overline{x F}\right],
$$

where $F$ is the total force, the sum of the external plus the space-charge force. When the total force is iinear in $x$, the two terms on the right side of Eq. (2.10) cancel, and there is no rms-emittance growth. Both nonlinear forces and non-Liouvillian processes can affect the averages $\overline{x^{\prime} F}$ and $\overline{x F}$, to make the right side of Equation 2.10 nonzero, and cause rms-emittance growth. However, nonlinear forces will not cause emittance growth if the two terms in brackets cancel. This is what happens when the beam reaches an equilibrium state with a nonuniform density distribution where the space-charge force is nonlinear.

For linear external forces, the external force contribution to Eq. (2.10) cancels, leaving only the space-charge force, which is nonlinear unless the spatial density distribution is uniform. Then, the right side of the equation can be expressed in a form proportional to the rate of change of space-charge field energy, ${ }^{14}$ which depends on beam intensity and 
on the rate of change of the spatial-density distribution. This formulation has led to some useful analytic formulas for space-charge-induced emittance growth for beams injected into transport and accelerator channels, as will be discussed in more detail later.

Another effect associated with time-dependent phenomena can lead to apparent growth of rms emittance. When accelerator parameters vary with time (jitter), the beam centroids and ellipse parameters can fluctuate with time. If the beam variations are too fast for the measurement apparatus to resolve, this will appear as an enlarged phase-space area and an apparent increase in rms emittance as a result of the superposition of ellipse contours at different times. Examples include beam current variations caused by ion source fluctuation, mechanical vibration of $\mathrm{rf}$ cavity elements such as drift tubes, and excitation of higher-order cavity modes. The beam will be mismatched or missteered at certain times and, if nonlinear external forces act on the beam, these effects can result in real growth of rms emittance.

\section{Emittance}

\subsection{Emittance in the DC Injector}

The first accelerator subsystem in the $\mathrm{H}^{-}$injector system is the dc injector, which must perform the functions of beam formation in an $\mathrm{H}^{-}$ion source, followed by extraction, dc acceleration, and finally transport and matching into the first component of the linac, the RFQ.

Some useful scaling relations have been derived for ion sources. ${ }^{15,16}$ If the extracted ions have a Maxwellian velocity distribution, and the beam is spatially uniform, the intrinsic rms-normalized emittance in each transverse plane can be written nonrelativistically for an assumed wailst condition at the extraction aperture as

$$
\varepsilon_{n}=0.5 r \sqrt{k T / m c^{2}}
$$

The quantity $r$ is the extraction-aperture radius, $k T$ is the ion temperature, and $m c^{2}$ is the ion rest energy. The space-charge-limited ion current is obtained from the Child-Langmuir law as

$$
I(A)=0.00173 \pi V(k V)^{1.5}\left[\frac{r}{d}\right]^{2}\left[\frac{Z}{A}\right]^{0.5}
$$

where $V$ is the extraction voltage, $d$ is the effective extraction gap width, and $Z$ and $A$ are the charge and mass numbers $\left(Z=1\right.$, and $A=1$ for $\mathrm{H}^{-}$ions). Keller assumes the maximum value of $r / d$ is constrained by the extraction aberrations to about unity, and proposes an empirical electric breakdown limit of

$$
d(\mathrm{~cm})=0.0014 V(\mathrm{kV})^{1.5}
$$

Reiser uses these assumptions to derive a maximum phase-space density (current divided by rms-normalized emittance), which is independent of extraction voltage, and using our emittance definitions is giver by

$$
\frac{I}{\varepsilon_{n}}\left[\frac{A}{\mathrm{~mm}-\mathrm{mrad}}\right]=3.0 \sqrt{\frac{Z}{k T(\mathrm{eV})}} .
$$


If $k T$ represents the actual ion temperature, Eq. (3.1.4) can be considered as a theoretical upper limit for ion-source phase-space density. Generally, emittance is the quantity that is actually measured, and ion temperatures are often considered to be effective values that include emittance growth.

The emittance of the extracted beam ${ }^{17}$ is determined by three effects: 1) the ion temperature $k T, 2)$ beam-optics aberrations that produce nonlinear motion during the extraction process, and 3) time-dependent fluctuations in the plasma resulting in variations in current and other parameters. Which effects are dominant depends on the type of ion source and the specific design characteristics.

For a sufficiently stable and quiescient plasma, the emittance growth from fluctuations, especially current fluctuations, can be kept small. If this is not the case, the emittance will be enlarged as a result of the superposition of extracted emittances with different orientations at different currents. For surface-type ion sources, where the $\mathrm{H}^{-}$are mainly produced on a Cesium-coated cathode surface, bombarded by $\mathrm{H}^{+}$ions, large ion temperatures $k T>10 \mathrm{eV}$ are obtained. For volume-type sources where the $\mathrm{H}^{-}$ions are produced mainly by charge exchange of fast cathode-produced ions on $\mathrm{H}$ atoms, the ion temperatures can be $k T<0.5 \mathrm{eV}$. For the surface-type sources, if the extraction optics can be designed to minimize the effects of aberrations, the lowest measured emittance values are consistent with those calculated from the $\mathrm{H}^{-}$ion temperature at emission. For volume-type sources, where the intrinsic temperatures are low, the emittance is usually determined by the aberrations at extraction. From Eq. (3.1.1) we obtain estimates of phase space density of $0.95 \mathrm{~A} / \mathrm{mm}$-mrad for the surface-type source (assuming $k T=10$ $\mathrm{eV}$ ), and $4.2 \mathrm{~A} / \mathrm{mm}-\mathrm{mrad}$ (assuming $k T=0.5 \mathrm{eV}$ ) for the volume-type source.

As a numerical example of an operating source, we consider the circular aperture mag= netron source (surface-type source), designed by the Brookhaven group ${ }^{18}$ for injection into an RFQ. The reported values of $x$ - and $y$-ncrmalized $90 \%$ emittances are $\varepsilon_{n}(90 \%) \pi=0.94$ and $1.0 \pi \mathrm{mm}$-mrad at both $70 \mathrm{~mA}$ and $36 \mathrm{~mA}$. The fact that emittance is independent of current is consistent with t''e assumption that for this source the emittances are not determined by the intrinsic ion temperature but by other effects such as aberrations. Assuming the rms-normalized emittance equals $\varepsilon_{n}(90 \%) / 4.6$ (valid for a Gaussian phase-space density), and averaging over the $x$ - and $y$-planes, gives $\varepsilon \pi=0.21 \pi \mathrm{mm}$-mrad, and at $70 \mathrm{~mA} \mathrm{a}$ brightness $\mathrm{I} / \varepsilon_{n}=0.33 \mathrm{~A} / \mathrm{mm}$-mrad, which is about a factor of three lower than the theoretical limit of $0.95 \mathrm{~A} / \mathrm{mm}$-mrad estimated above. Eq. (3.1.1) gives an effective temperature of $k T=41 \mathrm{eV}$, compared with the estimated value of $k T>10 \mathrm{eV}$, consistent with the hypothesis that aberrations or other effects have increased the emittance.

After the beam is extracted from the source, it is transported in the low-energy beam transport (LEBT) section to the RFQ entrance, where a small-diameter, converging beam must be produced for a proper match. ${ }^{19}$ In general two design approaches have been considered: 1) magnetic focusing and space-charge neutralized (compensated) transport in a plasma formed by beam-induced ionization of the residual gas, and 2) electric focusing (which removes the background plasma) and transport with no neutralization. Both approaches have advantages and disadvantages, and most of the operational experience has been with space-charge neutralized transport.

In space-charge neutralized transport, the $\mathrm{H}^{-}$beam ionizes the residual gas, creating both electrons and positive ions. Initially, the space-charge potential of the beam drives the electrons out and attracts the positive cins. If the gas density is sufficiently high, the positive-ion charge can build up until the space-charge forces of the beam are canceled, with a characteristic time constant $\tau=1 / n \sigma \nu$ of typically about $50 \mu \mathrm{sec}$. A steady state "an result, where the positive-ion density slightly exceeds the beam density, leading to 
net focusing and a pinch effect. Although the space-ch.arge forces are more than canceled, external focusing is still required to counteract the natural tendency of the beam to diverge because of its finite emittance. This focusing is now provided by magnetic lenses, which are compatible with the presence of the neutralizing plasma because they do not sweep away the positive ions.

The advantage of space-charge-neutralized transport for control of the space-charge defocusing forces can be seen from the Kapchinskii-Vladimirskii (K-V) envelope equation for transport of a round beam,

$$
a^{\prime \prime}+k_{o}^{2} a-\varepsilon_{n} / \beta^{2} \gamma^{2} a^{3}-\frac{K}{4 a}=0
$$

where $a$ is the rms beam size, $k_{0}$ is a measure of the average focusing strength, and the dimensionless generalized perveance describing the space-charge force is

$$
K=\frac{q I}{2 \pi \varepsilon_{0} m c^{3}(\beta \gamma)^{3}} .
$$

The ratio of the space-charge to the emittance term is

$$
\rho=K\left[\frac{\beta \gamma a}{2 \varepsilon_{n}}\right]^{2} .
$$

The matched beam size in a uniform focusing channel is determined by the solution of Eq. (3.1.5) when $a^{\prime \prime}=0$, and is determined by the space-charge term when $\rho>1$ (space-charge dominated) and by the emittance term when $\rho<1$ (emittance dominated).

As an example, consider $50 \mathrm{keV}$ and $50 \mathrm{~mA}$ of $\mathrm{H}^{-}$beam with an rms-normalized emittance of $\varepsilon_{n} \pi=0.2 \pi \mathrm{mm}$-mrad. Assuming solenoid focusing in a smooth approximation with a magnetic field of $1 \mathrm{~T}$ and solenoids that occupy half the space, we assume that

$$
k_{0}=\frac{e B}{2 \sqrt{2} m c \beta \gamma}=11.3 \mathrm{~m}^{-1}
$$

where a $1 / \sqrt{2}$ accounts for the filling factor of the solenoids. With no neutralization the full space-charge force is present, and we obtain $K=0.0032$, a matched beam size of $a=2.6 \mathrm{~mm}$, and the ratio of the space-charge to emittance term is $\rho=13.5$. The beam is space-charge dominated and it will be more difficult to focus it to a small diameter for matching into an RFQ. An interesting quantity is the space-charge potential between the center and the edge of the beam, which is

$$
\Delta \phi=\frac{I}{4 \pi \varepsilon_{0} c \beta}=150 \mathrm{~V} .
$$

For space-charge-neutralized transport the steady-state space-charge potential is not zero but is estimated as $\Delta_{\phi}=k T_{e} / e$. The electron temperature can be estimated ${ }^{20}$ from the relation $k T_{e} / e=0.67\left[V_{b} V i /\left(M_{b} / M_{e}\right)\right]^{1 / 2}$, where $V_{b}$ is the beam energy, $V_{i}$ is the ionization potential of the background gas, and $M_{b}$ and $M_{e}$ are the masses of the beam ions and electrons. Using the ionization potential of molecular hydrogen of $V_{i}=15.4 \mathrm{eV}$, we obtain $\mathrm{kT}_{e}=13.6 \mathrm{GV}$. From Eq. (3.1.9) the residual or effective current of $4.6 \mathrm{~mA}$ is obtained, with a corresponding effective perveance of $K=0.00029$. The $\mathrm{rms}$ beam size 
becomes $a=1.34 \mathrm{~mm}$, and the ratio $\rho=0.33$, ie. an emittance-dominated beam that is easier to focus into the RFQ.

Space-charge neutralization is easy to implement, by simply increasing the gas pressure, either by control of the pumping rate or by deliberately introducing a background gas. But there are significant disadvantages such as 1) difficulty in providing constant and uniform gas density, 2) stripping loss of the $\mathrm{H}^{-}$beam, and 3 ) instability and emittance growth from the associated plasma fluctuations. ${ }^{21}$

The dominant emittance growth mechanism for space-charge-neutralized transport, identified by Allison, ${ }^{22}$ is the variation of the output ellipse orientation caused by fluctuations in either the current from the ion source or the fraction of neutralization. As a result, the time-averaged phase-space area is increased, and this corresponds to an apparent growth of emittance. Allison quotes a frequency range of about 1 to $1000 \mathrm{kHz}$ for these fluctuations.

In addition to the fast current fluctuations, the amount of space-charge neutralization, the corresponding effective current, and the output phase-space ellipse parameters vary with time for a pulsed ion source. When the pulse length is small compared with the space-charge neutralization time constant $\tau$ (typically about $50 \mu \mathrm{sec}$ ), the output ellipse parameters correspond approximately to unneutralized (full space-charge) transport. For pulse lengths large compared to $\tau$, the average ellipse is dominated by the steady-state (space-charge neutralized transport). For pulse lengths near $\tau$, one can observe strong variations of tic ellipse parameters throughout the pulse as the beam approaches equilibrium. These time variations as the neutralization varies from 0 to near $100 \%$ have been measured. ${ }^{23}$ If the time resolution of the emittance measurement exceeds the pulse length, these variations result in apparent emittance growth. Nevertheless this effect causes a time-dependent beam mismatch at the entrance to the RFQ, which generally would result in time-dependent emittance growth within the RFQ.

The main emittance growth concern for the unneutralized transport with electrostatic focusing is from aberrations. ${ }^{24}$ The main problem area may be the final match into the RFQ, which requires a large beam within the final lens to obtain a small beam diameter inside the RFQ. A second source of potential emittance growth also occurs when the beam is expanded before final matching into the RFQ, and space-charge field energy is converted to thermal energy in the beam. ${ }^{25,14}$

\subsection{Emittance in the RF Linac}

The conventional high-current if ion-linac configuration begins with a dc injector and is followed by the racio-frequency quadrupole (RFQ) linac ${ }^{26,27}$ used to bunch and accelerate the beam from $100 \mathrm{keV}$ or below to a few MeV for protons. The RFQ uses if electric-quadrupole fields to provide strong focusing for low-velocity particles. To maintain the acceleration efficiency and transverse focusing at higher energies, the drift-tube linac (DTL) with magnetic quadrupole lenses within the drift tubes becomes a better choice. The RFQ-to-DTL transition can occur at a few $\mathrm{MeV}$ for a proton or $\mathrm{H}^{-}$beam. At much higher energies, the rf-pover efficiency of the DTL decreases below that of the coupledcavity linac (CCL) structures. ${ }^{28}$ The transition from DTL to CCL becomes attractive at about $100 \mathrm{MeV}$ for a proton linac.

The transverse emittance growth mechanisms in a high-intensity linac can be separated into three reneral categories: 1) effects in a perfect linac with aligned and rmsmatched beams, 2) effects for misaligned and rms-mismatched beams, and 3) effects associat $: d$ with misalignments and errors in the linac structure. 
When comparing emittance-growth effects, one should be cautious about making general conclusions as to which mechanisms are most important, because different factors can dominate in different accelerators. The primary sources of transverse emittance growth in a perfect linac with no misalignments and no field errors are generally from self forces and $\mathrm{rf}$ forces. Both forces are nonlinear, couple the degrees of freedom, and are the cause of emittance growth because the transverse oscillation frequencies depend on amplitude (nonlinearities), and on the other coordinates (coupling). A resulting decoherence of transverse oscillations for different particles causes distortion of the initial phase space, unless the distribution is already internally matched to these forces.

For high-current linacs we will be concerned with emittance growth resulting from self forces as the dominant effect. In general the self forces include both electric and magnetic forces. The latter are usually much smaller in a proton linac, so we will refer to these self forces just as space-charge forces. We begin by discussing space-charge-induced emittance growth with a qualitative discussion of beam matching into an accelerator channel. We distinguish between what we will call internal matching and rms matching. Internal matching constrains the 6-D phase-space distribution so that the isodensity contours coincide with the particle phase-space trajectories. For an internally matched beam the distribution will be in equilibrium in the accelerator channel, and no emitiance growth will occur, even though nonlinear forces may act on the beam. Such an equilibrium distribution is independent of time (stationary) if the focusing is uniform along the accelerator, or is periodic for a periodic-focusing channel. Examples of equilibrium distributions have been studied for 2-D transport channels. ${ }^{29}$ The most frequently studied is the $\mathrm{K}-\mathrm{V}$ distribution, which, unfortunately is physically unrealistic because the beam is distributed on the surface of a hyperellipsoid in 4-D phase space, resulting in no particles in the central core of the 4-D space. This distribution results in uniform ellipses for all 2-D projections. ${ }^{30}$

Given a beam that is not internally matched, one would like to be able to transform it into an internally matched, equilibrium distribution for the given accelerator channel, without increasing the rms emittance in the process. We do not know whether it is even possible in principle to perform such a transformation, without accompanying emittance growth. Nevertheless, it is feasible to match the rms beam sizes in each degree of freedom. This is accomplished by providing a beam-optics transformation, so that the rms sizes are exactly right to balance the sum of the focusing, space-charge, and emittance terms in the rms-envelope equations. In a uniform focusing channel the matched rms sizes are then constant. In a periodic channel the rms sizes will undergo a periodic flutter about their average values. An rms-matched beam is not necessarily internally matched. If it is not, the beam distribution is not in equilibrium, and the beam will evolve with the possibility of irreversible emittance growth. Nevertheless, rms matching is an important characteristic of an internally matched equilibrium distribution, and injection of an rms-matched beam can be considered a first approximation to providing the desired internally matched beam.

Numerical simulations of nonequilibrium linac beams show that such beams do evolve to quasi-equilibrium distributions, which change only slowly as the beam is aicelerated. The evolution of the beams is usually accompanied by rms-emittance change as a result of both nonlinearity and coupling between degrees of freedom. Experience has shown that the velocity distributions of the final beams are Maxwellian-like and when focusing is linear, the spatial distribution of a space-charge-dominated beam consists of an approximately uniform charge-density core of density $n$. The density decreases to zero over a finite distance approximately equal to the Debye length $\lambda_{D}$ given nonrelativistically by Eq. (2.2). For space-charge-dominated beams $\lambda_{D} \ll a$, and the equilibrium spatial distribution is approximately uniform with a sharp falloff at the edges. For emittance-dominated beams 
$\lambda_{D} \gg a$, and the Debye tail occupies essentially the entire spatial extent of the beam, resulting in a peaked Gaussian-like charge density. Among 2-D continuous equilibrium beams, the $\mathrm{K}-\mathrm{V}$ distribution is anomalous because it is always uniform in its projection to real space, regardless of the relative importance of emittance and space charge. However, this dist ribution does not correspond to the final equilibrium state of beams observed in numerical simulation studies.

It is further observed in numerical simulation that the emittance growth of beams that evolve to a final equilibrium distribution is associated mostly with a halo of lowdensity particles in phase space. This halo is especially undesirable for high-duty-factor linacs because it results in particle losses on the accelerator walls and radioactivation of the accelerator.

In early emittance growth studies ${ }^{31,32}$ of error-free, high-current proton drift-tube linacs, it was concluded that emittance growth caused by the transverse of forces was small. Nonlinear space-charge forces, associated with longitudinal to transverse coupling, were identifed as the primary source of the observed transverse emittance growth. Chasman also found that this emittance-growth mechanism leads to a lower limit for the output emittance as input emittance is decreased at fixed beam current. Later studies ${ }^{33}$ showed that emittance growth from longitudinal to transverse coupling couid be physically correlated with the dependence of the transverse oscillation frequency on the longitudinal position of the particles in the bunch. Furthermore, the results were not very sensitive to the input particle distribution.

An observation of longitudinal emittance decrease associated with the transverse emittance increase led to the suggestion of equipartioning. ${ }^{34}$ In this picture the emittance changes are the result of the evolution of a high-current beam towards an equilibrium distribution in which approximate kinetic-energy balance in the center of mass, or equipartioning, would be established. This suggests that if the input beam could be equipartioned in the accelerator in addition to being $\mathrm{rms}$ matched, there would result a better approximation to the ideal internally matched beam, and therefore space-charge induced emittance growth would be minimized.

Equipartitioning would mean that the mean-square center-of-mass velocities in each degree of freedom would be equal. This corresponds to the condition that

$$
\sigma_{x} \varepsilon_{x}=\sigma_{y} \varepsilon_{y}=\sigma_{z} \varepsilon_{z}
$$

where $\sigma_{x}, \sigma_{y}$, and $\sigma_{z}$ are phase advances per focusing period, associated with both the focusing and the space-charge forces. For a given set of input beam emittances, $\varepsilon_{x}, \varepsilon_{y}$, and $\hat{\varepsilon}_{z}$, and a given beam intensity, the condition given by Eq. (3.2.1) imposes a constraint on the relative focusing forces in the three degrees of freedom of the accelerator channel. Thus, while rms matching is achieved by providing a suitable beam-optical matching section before the beam is injected into the linac, the equipartitioning condition depends both on the input beam, through the beam curres.. aid the emittances, and the accelerator design, which determines the focusing and the phase advan! os.

Additional understanding of the equipartitioning dynamics was obtained from the work of Hofmann, ${ }^{35}$ who identified the longitudinal-to-transverse space-charge coupling effects with coherent coupling instabilities associated with anisotropy in the beam. Underlying this approach is a particular point of view for the description of the emittance growth. ${ }^{36}$ The idea is that the emittance growth is the result of the excitation of unstable collective modes of oscillation of the beam. The beam is characterized by an infinite number of eigenmodes of collective oscillation. For example, a dipole mode corresponds to the displacement of the centroid and coherent oscillations of the whole beam about the axis as 
a result of the external focusing forces. Also there are higher-order modes, some of which are internal, involving radial and azimuthal (quadrupole, sextupole, etc.) density oscillations of the beam. The first reported study of such modes for a 2-D round $K-V$ distribution in a uniform focusing channel was made by Gluckstern, ${ }^{37}$ who identified many modes and derived their stability characteristics. Studies for the K-V beam in a quadrupole channel have also been made. ${ }^{38}$ Some of the modes were found to be unstable at a certain threshold values of intensity. Numerical simulation is always required to determine whether an unstable mode produces emittance growth before the beam stabilizes.

The existence of unstable collective modes with different intensity thresholds suggests that it is predominantly through these instabilities involving the internal rearrangement of the beam distribution that the nonlinear space-charge forces cause emittance growth. ${ }^{36}$ This picture is useful if it can be determined which modes are easily excited and accompanied by significant emittance growth, and which are relatively harmless and lead to redistribution of charge without much growth.

With this background in mind, Hofmann studied the K-V distribution with an asymmetry between the $x$ - and $y$-planes and derived the instability thresholds for the different modes. Although the studies correspond to continuous beams in an $x$ - $y$ geometry, Hofmann found that the same instability thresholds were approximately valid for the $r-z$ geometry of a bunched beam. It was found that equipartitioned beams were stable with respect to these instabilities, and that generally the requirement for avoiding emittance growth even allowed some relaxation of exact equipartitioning. The predictions of Hofmann's model were further tested for high-current beams in drift-tube linacs with constant accelerating fields by Jameson, ${ }^{39}$ who confirmed that equipartitioned input beams produced the minimum emittance growth, while non-equipartitioned beams could produce a significant transfer of energy and emittance between the longitudinal and transverse planes. Jameson showed from simulation how the parameters of the non-equipartitioned accelerating beam can change in the space defined by the mode-stability plots derived by Hofmann. ${ }^{35}$ This behavior can be complicated and makes it difficult to derive simple design guidelines for avoidance of emittance growth. Perhaps the simplest design approach is to require exact equipartitioning as defined by Eq. (3.2.1). A less restrictive guideline is suggested by Hofmann, whose conservative criterion is that energy anisotropy is generally tolerable when the phase advance ratio $\sigma_{l} / \sigma_{t}<1$ where $\sigma_{l}$ and $\sigma_{t}$ are the phase advances for longitudinal and transverse motion respectively. The growth times from numerical simulation were typically about 1 to 2 transverse oscillation periods.

Later, a second space-charge-induced emittance-growth mechanism was discovered ${ }^{25,14}$ that even affects beams that are both $\mathrm{rms}$ matched and symmetric or equipartitioned, but are internally mismatched. This mechanism has been called charge redistribution. When a beam is injected into a transport or accelerator channel, the charged beam particles, behaving like a plasma, adjust their positions to shield the external fields from the interior of the beam. For linear external fields in the extreme space-charge (zero-emittance) limit, this implies a charge rearrangement to a uniform density to produce a linear space-charge field for exact shielding. Finite emittance beams are observed in numerical simulation to evolve to an internally matched charge density with a central uniform core and a finite thickness boundary, whose width is about equal to the Debye length. The rms-emittance growth arises from the nonlinear space-charge fields while the beam has nonuniform density and is undergoing internal plasma oscillations. The emittance growth can also be described as the result of the decoherence of the plasma oscillation phai es for particles with different amplitudes, resulting in distortion of the phase-space area. This mechanism of emittance growth has the smallest known growth time; the full emittance growth occurs during only 
one-quarter of a plasma period, followed by damped oscillations of the emittance for typically ten or so additional plasma periods. In a high-current linac the full growth can occur within a single cell. This mechanism can become important when beams that have been internally matched to very strong focusing channels are injected after rms matching into weaker focusing channels. In the strong focusing channel where the matched beam size is small, the emittance term in the envelope equation dominates over the space-charge term, and the equilibrium distribution has a strongly peaked, spatial density, generally having the appearance of a. Saissian distribution. In the weak focusing channel the rms beam size is large, the snuce-charge term dominates over the emittance term, and the corresponding equilibrium distribution is nearly uniform.

If the rms-matched input beam has the peaked spatial profile characteristic of the strong focusing channel, the beam density will change from peaked to nearly uniform in the weak focusing channel, and the change in shape of the distribution can be used to calculate the emittance growth. This results from the fact that, for a fixed rms beam size, the space-charge field energy is minimum for a uniform beam and increases as beams become more nonuniform. The evolution of the beam from peaked to uniform is accompanied by conversion of space-charge field energy to center-of-mass kinetic energy, which causes an increase in temperature and emittance. The emittance growth for a spherical bunch containing $N$ particles, each with charge $q$, is obtained from the expression ${ }^{40}$

$$
\frac{\varepsilon_{n, f}}{\varepsilon_{n, i}}=\left[1+q^{2} N a U_{n i} / 60 \sqrt{5} \pi \varepsilon_{0} \gamma^{3} m c^{2} \varepsilon_{n, i}^{2}\right]^{1 / 2}
$$

where $a$ is the rms beam size and $U_{n i}$ is the initial nonlinear field-energy parameter, a function only of the shape of the initial distribution. Emittance growth from charge redistribution is sensitive to the initial spatial charge density. For an initial Gaussian profile, $U_{n i}=0.308$, whereas for a uniform density $U_{n i}=0.0$.

To avoid emittance growth from charge redistribution it is necessary either to avoid transitions to accelerator channels with weaker focusing, or always to provide input beams with spatial profiles that are as uniform as possible. Other guidelines for minimizing emittance growth from charge redistribution can be inferred from Eq. (3.2.2). For a given beam current $I$, defined as the average value over an if period, the number $N$ of particles per bunch is given by $N=I \lambda / q c$, where $\lambda$ is the $\mathrm{rf}$ wavelength. Equation (3.2.2) predicts that the emittance growth is less at high frequencies, a result that appears because a high frequency linac has less charge per bunch for a given current. This condition was first reported $^{33}$ in studies of emittance growth that probably included equipartitioning effects, and is more generally valid than for the charge-redistribution eñect alone. Equation (3.2.2) also shows that larger beams are more susceptible to increased emittance growth from nonlinear space-charge forces.

Numerical simulation studies of transverse emittance growth in an RFQ linac have also been reported. ${ }^{41}$ The main features are: 1) the emittance growth is predominantly caused by space-charge forces, 2) most of the growth occurs while bunching the beam and is a strong function of the longitudinal beam size, 3 ) the growth is weakly dependent on the beam current, 4) above a certain current the growth is almost independent of the initial distribution, and 5) as for the drift-tube linac the final emittance approaches a lower limit as the initial emittance approaches zero at fixed beam current. The emittance growth in the RFQ bunching section may be a combination of the equipartitioning effect and charge redistribution as the bunching forces increase the peak value of the beam current and drive the beam into a more space-charge-dominated regime. A semi-empirical emittance 
growth formula was obtained ${ }^{41}$ based on Eq. (3.2.2), which is in good agreement with the numerical simulation results. This formula shows the advantage of high frequency and strong focusing for control of space-charge-induced emittance growth in the RFQ.

Next we discuss the additional emittance growth experienced by beams that are misaligned or rms mismatched. Misalignment results in a beam which undergoes coherent oscillations about the axis. These oscillations produce emittance growth if there are external forces that are nonlinear or couple the degrees of freedom. Then the misalignments put the beam into regions where the nonlinearities and coupling terms have a greater effect on the motion. Both nonlinearity and coupling are characteristic properties of the transverse rf-defocusing forces in the rf gaps, and therefore in practice additional emittance growth does result from beam misalignment. Consequently, particles at different transverse distances from the axis, and with different values of the longitudinal coordinate or phase, rotate in phase space at different frequencies. An initial ellipse eventually becomes distorted, resulting in an increase in rms emittance. Space-charge forces do not contribute to additional emittance growth for misaligned beams. Forces from image charges induced on the walls by the beam are nonlinear and could produce emittance growth. These effects are generally small for aligned beams in proton linacs, but for misaligned beams can lead to growth. ${ }^{42}$

If the beam is only rms mismatched, it is centered on the beam axis, but the initial shape and orientation of the ellipse are not the same as for the matched beam at low intensity. The rms beam envelope oscillates as the mismatched ellipse rotates in phase space. For a space-charge-dominated beam the mismatch oscillations are plasma oscillations. Additional potential energy becomes available for emittance growth of mismatched beams, which means that rms matching of beams is very important for the control of emittance growth. ${ }^{43,44}$

A significant source of emittance growth from beam instability is associated with a beam in a periodic channel. This is caused by the envelope instability, ${ }^{38,45}$ which occurs as a result of excitation of coherent modes by the periodic focusing structure. Some of the modes have nonuniform density distribution, whose nonlinear fields can produce significant emittance growth. The safest course to avoid the resonances is to design the accelerator channel at a zero current phase advance per focusing period, no larger than $\sigma_{0}=\pi / 2$.

The final general category of emittance growth effects is the effect of linac errors. Errors of transverse misalignment are often of most concern. They cause steering of the beam from the nominal beam axis, and result in the same kind of emittance growth effects as for a misaligned beam. The real cause of emittance growth is not the misalignments but the increased nonlinearity in the transverse rf fields experienced by the misaligned beams. In longitudinal space the corresponding effect is tank $\mathrm{rf}$-phasing errors, which can increase the phase excursions of the beam, and contribute to transverse emittance growth because of the longitudinal-to-transverse coupling. Quadrupole gradient errors produce rms mismatch in the beam, which increases the particle amplitudes. RF phase and amplitude errors will produce a corresponding longitudinal mismatch, which likewise can affect the transverse emittance because of the coupling.

Another source of error-induced emittance growth comes from quadrupole rotation about the longitudinal axis, which causes coupling of $x$ - and $y$-motions. Other effects, such as higher (nonlinear) magnetic multipoles, some of which are magnet-fringe fields, are usually smaller in linacs. 


\subsection{Emittance in Synchrotron Injector Rings}

Some important emittance growth mechanisms in the injector rings include the following. ${ }^{46}$

a) Mismatch of either the centroid or rms amplitude at injection into the ring, which results in larger excursions of the bearm into regions of nonlinear fields.

b) Multiple Coulomb scattering in the stripper foil during the injection process of the $\mathrm{H}^{-}$ beam.

c) Coherent transverse instabilities, which if uncorrected result in larger beam displacements and nonlinear fields.

d) Increase of emittance from momentum dispersion because of the dependence of the ellipse orientation on momentum.

e) Nonlinear space-charge-induced tune spread into resonance stop bands, which causes the tunes of beam particles repeatedly to cross stopbands of strong resonances as the perticles undergo transverse and longitudinal oscillations about the center of the bunch. The resulting growth of betatron amplitudes exposes the beam particles to nonlinear fields, which causes the emittance to grow.

The space-charge tune-spread mechanism is important at high intensity and is unique to ircular accelerators. The resonances and nonlinear fields are caused by imperfections in the dipole and quadrupole magnets, which form the focusing lattice. These imperfections can result from field errors and misalignments, and the real field distribution can be described as an infinite series of multipoles (dipole, quadrupole, sextupole, octupole, etc.). Multipole terms higher than quadrupole describe the nonlinear fields and contribute mostly at the larger displacements from the design orbit. Each of the multipole terms can be further decomposed into Fourier components in the azimuthal coordinate around the ring. ${ }^{4,48}$ Each Fourier component of a given multipole can drive the beam particles into resonance at certain values of the particle tunes, resulting in growth of the arnplitudes, which can eventually result in loss of the beam on the apertures. Dipole or first-order resonances drive the beam at integer tunes, quadrupole or second-order resonances at half-integer tunes, sextupole or third-order resonances at third-integer tunes, octupole or fourth-order resonances at quarter-integer tunes, etc. The particle tune does not have to lie exactly at the resonance value to be under the influence of the resonance; there is a stopband width within which a beam particle becomes locked into the resonance and will be driven to larger amplitudes.

In the Fermilab booster ring the space-charge tune-shift mechanism is believed to be the main source of intensity-dependent growth. ${ }^{6}$ Two competing effects occur after the full beam has been injected. The beam is adiabatically bunched before acceleration to achieve high capture efficiency, resulting in increased tune shift and tune spread becaise of the reduced bunching factor in the tune-shift formula Eq. (1.4). Transverse emittance growth occurs mostly during this time of maximum tune spread, and particle losses can also result because of the growth in amplitudes driven by resonances whose stopbands lie within the increased range of particle tunes. A second and beneficial effect is acceleration, which increases the $\beta \gamma^{2}$ factor in the tune-shift formula and, as the energy increases, the tune spread is eventually reduced below a level where significant resonances are excited.

The transverse emittance growth occurs because of the nonlinear forces that act on the particles, especiallv when their amplitudes are large. The resonant forces drive the particle amplitudes into the regions of stronger nonlinear fields. As can be seen from the tune-shift formula, the emittance growth reduces the tune spread, and emittance growth continues until either the particle tunes are safely outside the resonant stopbands or 
sufficient beam is lost on the apertures. For a given accelerator at a given choice of transverse zero-current tunes, there will be a characteristic maximum acceptable tune spread, determined by the operating point and strengths of the nearby stopbands. In the Fermilab booster the experimental relationship between emittance and intensity is linear (in accordance with the tune-shift formula.) with a slope that corresponds to a limiting space-charge tune shift of $\delta \nu=-0.38$.

Inspection of the tune-shift formula suggests several ways of reducing the emittance growth. One way is to reduce the tune spread by increasing the injection energy, and this approach is the main motivation for the project to upgrade the Fermilab linac from 200 to $400 \mathrm{MeV} .{ }^{49}$ The tune-spread limit can be increased by reducing the strengths of the nearby resonances. This can be accomplished by introducing correction magnets of the proper multipole content and azimuthal Fourier component to compensate for the strength of the given stopband. This approach has been successfully implemented for the CERN synchrotror booster, ${ }^{7}$ and a very large tune-shift value of $\Delta \nu=-0.60$ was achieved after compensating for one second-order and three third-order stopbands. Correction dipoles are routinely used to center the orbit, which reduces the strength of first-order stop-bands and also keeps the beam in the more linear-field region of the accelerator.

If a certain tune-spread limit is in effect for a given accelerator at a given operating point, it is important to know what will happen to an injected beam with too small an emittance (an emittance which results in too large a tune spread). The experience with the Fermilab booster, descri'ued above, suggests that the emittance will grow and the tune spread will decrease unti? the uncompensated stopbands are outside the tune spread of the beam. The emittance growth within the ring appears to provide a natural stabilizing mechanism, so external methods of spoiling the emittance before injection are unnecessary. However, the increased emittance is not necessarily desirable, because it results in reduced luminosity for the collider.

\section{Performance of High Brightness Injectors}

Having reviewed transverse emittance growth mechanisms in dc injectors, linacs, and synchrotrons, we now follow the evolution of transverse emittance of the proton beam through the world's highest energy accelerator system, at Fermilab. We recommend an earlier article by Finley ${ }^{50}$ as an excellent source of material on this subject for the Fermilab $\bar{p} p$ Tevatron collider facility. Table I shows the results of transverse emittance measurements on the proton beam at different locations and energies at Fermilab. ${ }^{50}$ 
Table I.

Summary of Measured Transverse RMS-Normalized Emittances in the Fermilab Accelerator Chain Given as $\pi \varepsilon_{n}(\mathrm{~mm} \cdot \mathrm{mrad})$

\begin{tabular}{llll}
\hline Location & $\begin{array}{c}\text { Energy } \\
(\mathrm{GeV})\end{array}$ & Horizontal & \multicolumn{1}{c}{ Vertical } \\
\hline Linac In & 0.00075 & $0.46 \pi$ & $0.57 \pi$ \\
Linac Out & 0.2 & $1.1 \pi$ & $1.1 \pi$ \\
Booster In & 0.2 & $2.5 \pi$ & \\
Booster Out & 8 & $2.5 \pi$ & \\
8-GeV Line & 8 & $1.7 \pi$ to $2.5 \pi$ & $1.7 \pi$ \\
8-GeV Main Ring & 8 & $1.7 \pi$ to $2.5 \pi$ & $1.3 \pi$ to $2.0 \pi$ \\
Main Ring & & & \\
$\quad$ Accelerated & 150 & $2.8 \pi$ & \\
Main Ring & & & \\
$\quad$ Coalesced & 150 & $4.5 \pi$ & \\
Tevatron & & & \\
$\quad$ Collider & 800 & $5 \pi$ to $10 \pi$ & $3.3 \pi$ to $5.0 \pi$ \\
\hline
\end{tabular}

The emittances in the rings quoted in Finley's article are the 6-rms values, and for the linac are $90 \%$ values. In Table I we have divided the 6 -rms values by 6 and the $90 \%$ values by 4.6 to convert them to rms values. The 4.6 factor assumes Gaussian distributions. The overall emittance growth factor from the linac input to full energy ranges from 10 to 20 in the horizontal plane and ranges from 6 to 9 in the vertical plane.

Table II shows the design rms-normalized emittances for the SSC accelerator chain. It shows an overall growth factor of only 5 , and a final emittance lower by a factor of 5 to 10 than the Fermilab value in the horizontal plane and lower by a factor of 3 to 5 in the vertical plane. This improvement in beam quality is a significant challenge which will require careful alignment and matching throughout the accelerator chain, and sufficient diagnostics to understand the beam characteristics at every stage.

Table II.

Surnmary of Design Transverse RMS-Normalized

Emittances in the SSC Accelerator Chain.

\begin{tabular}{llc}
\hline Location & $\begin{array}{c}\text { Energy } \\
(\mathrm{GeV})\end{array}$ & $\begin{array}{c}\pi \varepsilon_{n, r m s} \\
(\mathrm{~mm} \cdot \mathrm{mmrad})\end{array}$ \\
\hline Linac In & 0.000035 & 0.2 \\
Linac Out & 0.6 & 0.4 \\
LEB Out & 11 & 0.6 \\
MEB Out & 200 & 0.7 \\
HEB Out & 2000 & 0.8 \\
Collider & 20000 & 1.0 \\
\hline
\end{tabular}




\section{Acknowledgements}

material.

I wish to thank Martin Reiser for valuable suggestions and comments on this

\section{References}

1. Helen T. Edwards, "SSC Design Status," Part. Accel. 26 (1990) 151.

2. C. Ankenbrandt, C. Curtis, C. Hojvat, R. R. Johnson, C. Owen, C. Schmidt, L. Teng, and R. C. Webber, "H- Charge Exchange Systems," Proc. 11th Int. Conf. on HighEnergy Accelerators, Geneva, July 1990, p. 260.

3. M. K. Craddock, "High-Intensity Circular Proton Accelerators," High-Brightness Accelerators, NATO ASI Series B: Physics 178 (1986) 43.

4. L. J. Laslett, "On Intensity Limitations Imposed by Transverse Space-Charge Effects in Circular Particle Accelerators," Proc. 1963 Summer Study on Storage Rings, Accelerators, and Experimentation at Super-High Energies, BNL-7534, p. 325.

5. K. H. Reich, K. Schindl, and H. Schonauer, "An Approach to the Design of SpaceCharge Limited High-Intensity Synchrotrons," Proc. 12th Int. Conf. on High-Energy Accelerators, Fermilab, 1982, p. 438.

6. C. Ankenbrandt r d S. D. Holmes, "Limits in the Transverse Phase-Space Density in the Fermilab Booster," Proc. 1987 IEEE Part. Accel. Conf., Washington, DC, March, 1987 , p. 1066.

7. G. Gelato, L. Magnani, N. Rasmussen, K. Schindl, and H. Schonauer, "Progress in Space-Charge Limited Machines: Four Times the Design Intensity in the CERN Proton Synchrotron Booster," Ibid., p. 1298.

8. J. D. Lawson, "The Physics of Charged Particle Beams," Clarendon Press, Oxford, 1977 , p. 124.

9. Claude Lejeune and Jean Aubert, "Emittance and Brightness: Definitions and Measurements", Adv. Electronics and Electron Physics, Suppl. 13A, Applied Charged Particle Optics (1980) p. 184.

10. J. D. Lawson, "The Physics of Charged Particle Beams," Clarendon Press, Oxford 1977, p. 207.

11. F. J. Sacherer, "RMS Envelope Equations with Space Charge," IEEE Trans. Nucl. Sci. 18 (1971) 1105.

12. P. M. Lapostolle, "Possible Emittance Increase Through Filamentation Due to Space Charge In Continuous Beams," IEEE Trans. Nucl. Sci. 18 (1971) 1101.

13. T. P. Wangler, "Energy and Emittance Relations from RMS Equations of Motion for a Continuous Beam with Azimuthal Symmetry," Los Alamos Group AT-1 Memo AT1:84-323, Oct., 1984.

14. T. P. Wangler, K. R. Crandall, R. S. Mills, and M. Reiser, "Relationship Between Field Energy and Rms Emittance in Intense Particle Beams," IEEE Trans. Nucl. Sci. 32 (1985) 2196.

15. M. Reiser, "Current and Phase-Space Density Limits of Ion Sources," Los Alamos Group AT-1 Memo AT-1:86-45, Feb., 1986.

16. R. Keller, "Brightness Limits for Ion Sources," High-Brightness Accelerators, NATO ASI Series B: Physics 178 (1986) 395. 
17. P. Allison, "Some Comments on Emittance of $\mathrm{H}^{-}$Ion Beams:, Proc. 4th Int. Symp. on Production and Neutralization of Negative Ions and Beams," AIP Conf. Proc. 158 (1986) 465.

18. J. G. Alessi, "A Circular Aperture Magnetron for Injection into an RFQ," Proc. 4th Int. Symp. on Production and Neutralization of Negative Ions and Beams, AIP Conf. Proc. 158 (1986) 419.

19. K. R. Crandall, R. H. Stokes, and T. P. Wangler, "RF Quadrupole Beam Dynamics Design Studies," Proc. 1979 Linear Accelerator Conf., BNL 51134, Sept. 1979, p. 205.

20. M. E. Rudd, Phys. Rev. A20 (1979), 787.

21. P. Allison, A. J. T. Holmes, A. F. Lietzki, J. H. Whealton, and L. Wright, "Panel Discussion: Transport of Negative Ion Beams," Proc. 4th Int. Symp. on Production and Neutralization of Negative Ions and Beams, AIP Conf. Proc. 158 (1986) 520.

22. P. Allison, "Emittance Growth Caused by Current Variations in a Beam Transport Channel," IEEE Trans. Nucl. Sci. NS-32, (1985) 2556.

23. D. S. Barton, "Negative Ion Source Tests for $\mathrm{H}^{-}$Injection at the Brookhaven AGS," IEEE Trans. Nucl. Sci. NS-28 (1981) 2681.

24. O. A. Anderson, C. F. Chan, W. S. Cooper, W. B. Kunkel, J. W. Kwan, A. F. Lietzke, C. A. Matuk, P. Purgolis, and L. Soroka, "Design of a $200 \mathrm{~mA} \mathrm{DC} \mathrm{H^{- }}$ Injuztor for an RFQ," Proc. 1987 IEEE Part. Accel. Conf., Washington, DC, 1987, p. 289.

25. J. Struckmeier, J.Klabunde, and M. Reiser, Part. Accel. 15 (1984) 47.

26. I. M. Kapchinskij and V. A. Tepliakov, "Linear Ion Accelerator with Spatially Homogeneous Strong Focusing," Prib. Tekh. Eksp. 2 (1970) 19.

27. Richard H. Stokes and Thomas P. Warıler, "Radio-Frequency Quadrupole Accelerators and Their Applications," Ann. Rev. of Nucl. Sci. 38 (1988) 97.

28. S. O. Schriber, "High-Beta Linac Structures," Proc. 10th Linear Accelerator Conf., BNL 51134, 1980, p. 164.

29. I. M. Kapchinskij, "Theory of Resonance Linear Accelerators," Harwood Academic Publishers, 1985, p. 273.

30. I. M. Kapchinskij and V. V. Vladimirskij, "Limitations of Proton Beam Current in a Strong Focusing Linear Accelerator Associated with the Beam Space Charge," Proc. Int. Conf. on High-Energy Accelerators and Instrumentation, CERN, Geneva, 1959, p. 274.

31. R. Chasman, "Numerical Calculations of the Effects of Space Charge on Six Dimensional Beam Dynamics in Proton Linear Accelerators," Proc. 1968 Proton Linear Accelerator Conf., BNL 50120, 1968, p. 372.

32. R. Chasman, "Numerical Calculations on Transverse Emittance Growth in Bright Linac Beams," IEEE Trans. Nucl. Sci. NS-16 (1969) 202.

33. J. W. Staples and R. A. Jameson, "Possible Lower Limit to Linac Emittance", IEEE Trans. Nucl. Sci. NS-26, (1979) 3698.

34. P. M. Lapostolle, "Round Table Discussion of Space Charge and Related Effects," Proc. 1968 Proton Linear Accelerator Conf., BNL 50120, 1968, p. 437.

35. I. Hofmann and I. Bozsik, "Computer Simulation of Longitudinal-Transverse Space Charge Effects in Bunched Beams," Proc. 1981 Linear Accelerator Conf., Los Alamos, LA-9234-C, 1982, p. 116.

36. I. Hofmann, "Emittance Growth of Ion Beams with Space Charge," Nucl. Instrum. Meth. 187 (1981) 281.

37. R. L. Gluckstern, "Oscillation Modes in Two Dimensional Beams," Proc. 1970 Linear Accelerator Conf., Fermilab, 1970, p. 811. 
38. I. Hofmann, L. J. Laslett, L. Smith, and I. Haber, "Stability of the KapchinskijVladimirsky (K-V) Distributions in Long Periodic Transport Systems," Part. Accel. 13 (1983) 145.

39. R. A. Jameson, "Equipartitioning in Linear Accelerators," Proc. 1981 Linear Accelerator Conf., Los Alamos, LA-9234-C, 1982, p. 125.

40. T. P. Wangler, K. R. Crandall, and R. S. Mills, "Emittance Growth from Charge Density Changes in High-Current Beams," Proc. Int. Symp. on Heavy Ion Fusion, Washington, DC, AIP Conf. Proc. 152 (1986) 166.

41. T. P. Wangler, R. S. Mills, and K. R. Crandall, "Emittance Growth in Intense Beams," Proc. 1987 Part. Accel. Conf., IEEE Cat. No. 87CH2387-9, 1987, p. 1006.

42. C. M. Celeta, "The Effect of Nonlinear Forces on Coherently Oscillating Space-Charge Dominated Beams," Ibid., p. 996.

43. M. Reiser, "Emittance Growth in Mismatched Space-Charge Dominated Beams," Submitted to 1991 IEEE Part. Accel. Conf., San Francisco.

44. A. Cucchetti and M. Reiser, and T. Wangler, "Simulation Studies of Emittance Growth in RMS Mismatched Beams," Ibid.

45. J. Struckmeier and M. Reiser, "Theoretical Studies of Envelope Oscillations and Instabilities of Mismatched Intense Charged Particle Beams in Periodic Focusing Channels and Particle Accelerators," Part. Accel. 14 (1984) 227.

46. "Site-Specific Conceptual Design of the Superconducting Super Collider," SSC Lab. Report SSC-SR-1051, June 1990, p. 254.

47. E. J. N. Wilson, "Circular Accelerators-Transverse," Physics of Particle Acceierators, Vol. 1, AIP Conf. Proc. 153 (1987) 4.

48. E. J. N. Wilson, "The Importance of Non-Linearities in Modern Proton Synchrotrons," Theoretical Aspects of the Behavior of Beams in Accelerators and Storage Rings, CERN 77-13, July 1977, p. 111.

49. D. E. Young and R. J. Noble, "400-MeV Upgrade for the Fermilab Linac," Proc. 14th Int. Conf. on High-Energy Accelerators, Part 1, Part. Accel. 26 (1990) 205.

50. D. Finley, "Emittance Through the Fermilab Accelerator Chain," Physics of Particle Accelerators, Vol. 2., AIP Conf. Proc. 184 Vol. 2, (1989) 1910. 

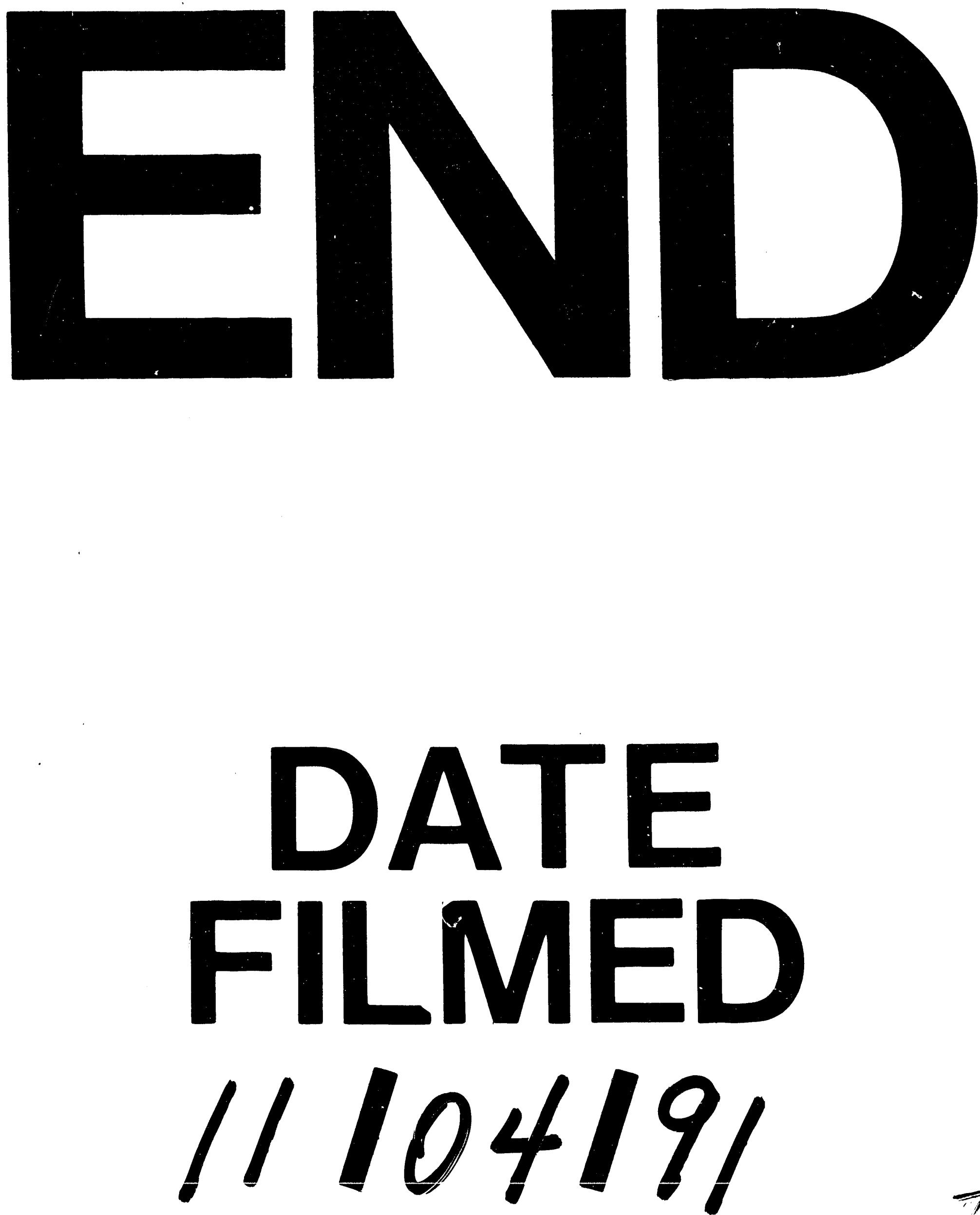

II 


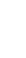

